\title{
An Iron and Copper System Catalyzed C-H Arylation of Azoles with Arylboronic Acids
}

\author{
Wei-Ye Hu \\ Pei-Pei Wang \\ Song-Lin Zhang* \\ Key Laboratory of Organic Synthesis of Jiangsu Province, Col- \\ lege of Chemistry, Chemical Engineering and Materials Science, \\ Soochow University, Suzhou 215123, P. R. of China \\ zhangsı@suda.edu.cn
}

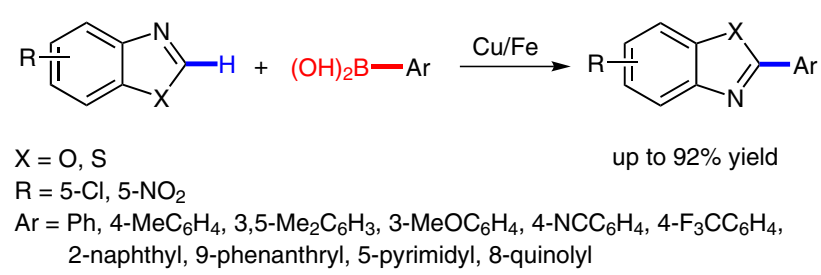

acids. ${ }^{10-12}$ But these protocols also suffered from problems, for example, low yields, narrow applied range, and high toxicity.

Considering the requirements of atom economy and environmental protection, the properties of copper and iron as catalysts have acquired great significance. ${ }^{13-15}$ We have recently demonstrated that an iron and copper system can catalyzed the formation of $\mathrm{C}-\mathrm{C}, \mathrm{C}-\mathrm{O}$, and $\mathrm{C}-\mathrm{N}$ bonds. ${ }^{16-19}$ As a part of our continuous efforts toward the development of an iron and copper system, we applied the iron-copper system to the reaction of azoles with arylboronic acids. In this paper, we report that an iron-copper catalyst system could very efficiently catalyze the cross-coupling between azole compounds and arylboronic acids with good yields.

The reaction of 1,3-benzoxazole (1a) with phenylboronic acid (2a) was used to optimize the reaction conditions. The results of our optimizations experiments for the copper salt, iron salt, base, ligand, oxidant, and solvent are given in Table 1. Initially, a copper salt was used as a single catalyst and a series of experiments were used to identify the most effective copper salt, base, ligand, oxidant, and solvent for the reaction. With all other conditions identically, copper(I) iodide gave the best result among other copper salts $[\mathrm{CuBr}$, $\mathrm{CuCl}, \mathrm{Cu}(\mathrm{OAc})_{2}, \mathrm{CuSO}_{4} \cdot 5 \mathrm{H}_{2} \mathrm{O}, \mathrm{Cu}(\mathrm{acac})_{2}$ ] (entries 1-6). Then, the base was varied (NaOMe, $\mathrm{K}_{2} \mathrm{CO}_{3}, \mathrm{KOAc}, \mathrm{KOt}-\mathrm{Bu}, \mathrm{Cs}_{2} \mathrm{CO}_{3}$ ) but none gave better results than lithium tert-butoxide (entries 7-11). From the experimental results, we could see that the ligand and oxidant also have an important influence on the reaction and that no product was obtained with certain ligand and oxidant combinations (entries 15, 16, 19, 20 , and 22). The best results were obtained using di-tertbutyl peroxide (DTBP) (entry 1 ). When clean oxidants, such as oxygen or hydrogen peroxide, were used, there was no improvement in the yield (entries 21 and 22). Next we investigated the effect of the ligand (Figure 1) and better results were obtained using 1,10-phenanthroline (L1) as the 
Table 1 Optimization of the Reaction Conditions ${ }^{\mathrm{a}}$

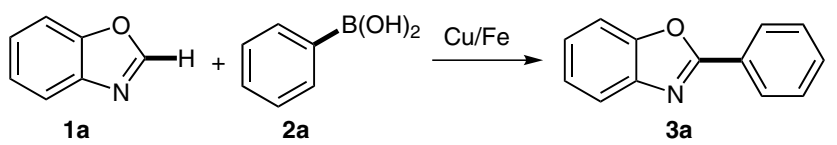

\begin{tabular}{|c|c|c|c|c|c|c|c|}
\hline Entry & Copper salt & Iron salt & Base & Ligand $^{\mathrm{b}}$ & Oxidant & Solvent & Yield ${ }^{c}(\%)$ \\
\hline 1 & Cul & - & LiOt-Bu & L1 & DTBP & DMSO & 50 \\
\hline 2 & $\mathrm{CuBr}$ & - & LiOt-Bu & L1 & DTBP & DMSO & 44 \\
\hline 3 & $\mathrm{CuCl}$ & - & LiOt-Bu & L1 & DTBP & DMSO & 42 \\
\hline 4 & $\mathrm{Cu}(\mathrm{OAc})_{2}$ & - & LiOt-Bu & L1 & DTBP & DMSO & 10 \\
\hline 5 & $\mathrm{CuSO}_{4} \cdot 5 \mathrm{H}_{2} \mathrm{O}$ & - & LiOt-Bu & L1 & DTBP & DMSO & 23 \\
\hline 6 & $\mathrm{Cu}(\mathrm{acac})_{2}$ & - & LiOt-Bu & L1 & DTBP & DMSO & 27 \\
\hline 7 & Cul & - & $\mathrm{NaOMe}$ & L1 & DTBP & DMSO & 16 \\
\hline 8 & Cul & - & $\mathrm{K}_{2} \mathrm{CO}_{3}$ & L1 & DTBP & DMSO & 13 \\
\hline 9 & Cul & - & KOAC & L1 & DTBP & DMSO & 27 \\
\hline 10 & Cul & - & KOt-Bu & L1 & DTBP & DMSO & 42 \\
\hline 11 & Cul & - & $\mathrm{Cs}_{2} \mathrm{CO}_{3}$ & L1 & DTBP & DMSO & 26 \\
\hline 12 & Cul & - & LiOt-Bu & L2 & DTBP & DMSO & 40 \\
\hline 13 & Cul & - & LiOt-Bu & L3 & DTBP & DMSO & 20 \\
\hline 14 & Cul & - & LiOt-Bu & L4 & DTBP & DMSO & 18 \\
\hline 15 & Cul & - & LiOt-Bu & L5 & DTBP & DMSO & 0 \\
\hline 16 & Cul & - & LiOt-Bu & L6 & DTBP & DMSO & 0 \\
\hline 17 & Cul & - & LiOt-Bu & L1 & $\mathrm{I}_{2}$ & DMSO & 24 \\
\hline 18 & Cul & - & LiOt-Bu & L1 & TBHP & DMSO & 31 \\
\hline 19 & Cul & - & LiOt-Bu & L1 & NBS & DMSO & 0 \\
\hline 20 & Cul & - & LiOt-Bu & L1 & DDQ & DMSO & 0 \\
\hline 21 & Cul & - & LiOt-Bu & L1 & $\mathrm{O}_{2}$ & DMSO & 40 \\
\hline 22 & Cul & - & LiOt-Bu & L1 & $\mathrm{H}_{2} \mathrm{O}_{2}$ & DMSO & 0 \\
\hline 23 & Cul & - & LiOt-Bu & L1 & DTBP & DMF & 44 \\
\hline 24 & Cul & - & LiOt-Bu & L1 & DTBP & NMP & 35 \\
\hline 25 & Cul & - & LiOt-Bu & $\mathbf{L 1}$ & DTBP & toluene & 52 \\
\hline 26 & Cul & - & LiOt-Bu & L1 & DTBP & benzene & 41 \\
\hline 27 & Cul & $\mathrm{Fe}_{2} \mathrm{O}_{3}$ & LiOt-Bu & $\mathbf{L 1}$ & DTBP & toluene & 87 \\
\hline 28 & Cul & $\mathrm{Fe}_{3} \mathrm{O}_{4}$ & LiOt-Bu & L1 & DTBP & toluene & 83 \\
\hline 29 & Cul & $\mathrm{FeCl}_{2}$ & LiOt-Bu & L1 & DTBP & toluene & 73 \\
\hline 30 & Cul & $\mathrm{FeCl}_{3}$ & LiOt-Bu & L1 & DTBP & toluene & 80 \\
\hline 31 & - & $\mathrm{Fe}_{2} \mathrm{O}_{3}$ & LiOt-Bu & L1 & DTBP & toluene & 0 \\
\hline 32 & Cul & $\mathrm{Fe}_{2} \mathrm{O}_{3}$ & LiOt-Bu & L1 & DTBP & toluene & $45^{d}$ \\
\hline 33 & Cul & $\mathrm{Fe}_{2} \mathrm{O}_{3}$ & LiOt-Bu & L1 & DTBP & toluene & $60^{\mathrm{e}}$ \\
\hline 34 & Cul & $\mathrm{Fe}_{2} \mathrm{O}_{3}$ & LiOt-Bu & L1 & - & toluene & $77^{f}$ \\
\hline
\end{tabular}

a Reaction conditions: 1 a $(0.5 \mathrm{mmol})$, phenylboronic acid (2a, 2 equiv), copper salt ( $20 \mathrm{~mol} \%)$, iron salt ( $20 \mathrm{~mol} \%)$, base ( 3 equiv), ligand (20\%), oxidant ( 2 equiv), solvent $(3 \mathrm{~mL}), 110^{\circ} \mathrm{C}, 12 \mathrm{~h}$.

${ }^{\mathrm{b}}$ Isolated yield based on 1a after silica gel chromatography.

c See Figure 1.

${ }^{d}$ Using 1.5 equiv of phenylboronic acid (2a).

e Using 1.7 equiv of phenylboronic acid (2a).

${ }^{\mathrm{f}}$ Using 2 equiv of $\mathrm{Fe}_{2} \mathrm{O}_{3}$. 
oxidant compared to TMEDA (L2), DMEDA (L3), and 2,2'-bipyridyl (L4) (entries 12-14). Therefore, 1,10-phenanthroline (L1) was selected as the optimal ligand for its catalytic effect and inexpensive price. Finally, the solvent was varied and toluene was found to be the best choice (entries 2326).
L1

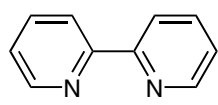

L4

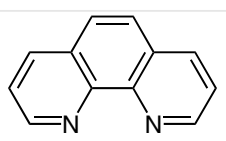

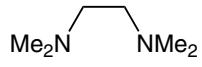

L2

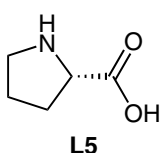

L5

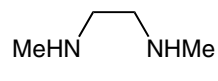

L3

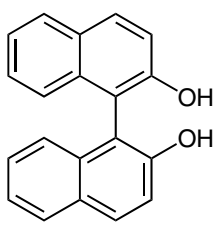

L6
Figure 1

Although a great number of experiments have been performed to improve the yield, we have not achieved our aim as the copper salt was the only catalyst. Interestingly, when an iron salt was added to work jointly with the copper salt, the yield rose sharply (entries 27 and 28). From the results, we could see that the iron salt is indispensable for a high yield and iron(III) oxide was the best from several common iron salts $\left(\mathrm{Fe}_{2} \mathrm{O}_{3}, \mathrm{Fe}_{3} \mathrm{O}_{4}, \mathrm{FeCl}_{2}, \mathrm{FeCl}_{3}\right)$ (entries 27-30).

The highest yield was obtained using the conditions in entry 27 and several experiments were performed varying the proportions of the reactants. We have reason to believe that the iron salt functions as a pre-oxidant. The reaction was carried out solely using the iron salt to ascertain whether the copper salt and iron salt were working jointly or separately; no product was obtained in the absence of the copper salt (entry 31). It follows that iron salt and copper salt are a collaborative catalyst in the reaction. The reaction with iron(III) oxide (2 equiv) in the absence of di-tertbutyl peroxide gave the product in $77 \%$ yield (entry 34 ). Reducing the amount of phenylboronic acid gave the product in lower yields (entries 32 and 33). We selected copper(I) iodide $(20 \% \mathrm{mmol})$, iron(III) oxide $(20 \% \mathrm{mmol})$, lithium tertbutoxide (3.0 equiv), 1,10 -phenanthroline $(20 \% \mathrm{mmol})$, ditert-butyl peroxide ( 2.0 equiv), and toluene $(3.0 \mathrm{~mL})$ as the optimal reaction conditions.

With the optimized reaction conditions in hand, we next explored the substrate scope of the reactions of diversified azoles with arylboronic acids. To evaluate the generality of this reaction, 1,3-benzoxazole (1a) was reacted with a range of arylboronic acids and the results are summarized in Table 2. The results show that 1,3-benzoxazole reacts well with arylboronic acids. Electron-poor or -rich arylboronic acids containing functionalities such as methyl, methoxy, cyano, or trifluoromethyl were studied and pyrimidine or quinoline rings as well. The results of the reactions of 4methylphenylboronic acid (2b) and 3,5-dimethylphenylboronic acid (2c) show that electron-donating groups may reduce the yield of the products (entries 2-4); the methoxy group affects the yield with a stronger electron-donating ability than methyl. When arylboronic acids linked with electron-withdrawing groups in the para position, such as 4-(trifluoromethyl)phenylboronic acid (2f) and 4-cyanophenylboronic acid $(\mathbf{2 e})$, were used, the yields of products dropped sharply (entries 5 and 6). Polycyclic arylboronic acid like naphthalen-2-ylboronic acid (2g) and phenanthren-9-ylboronic acid $(\mathbf{2 h})$ also reacted with 1,3-benzoxazole to provide the products $\mathbf{3 g}$ and $\mathbf{3 h}$ in $80 \%$ and $63 \%$ yields, respectively (entries 7 and 8). Pyrimidin-5-yl- or quinolin-8-yl-substituted boronic acids gave the products 3j and 3k, respectively, in acceptable yields (entries 10 and 11). The optimized reaction conditions were also applicable in the reactions of 5-chloro-1,3-benzoxazole (1) $), 5$-nitro1,3-benzoxazole (1c), and 4-phenyloxazole (1d) with arylboronic acids (entries 12-16).

Table 2 Reaction of Oxazoles with Arylboronic Acids

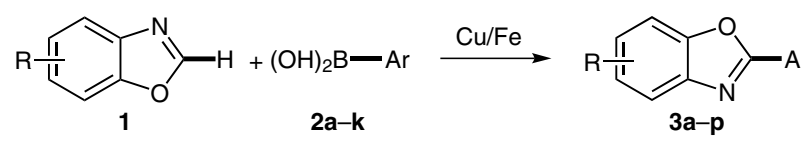

\begin{tabular}{|c|c|c|c|c|}
\hline Entry & $\mathrm{R}$ & $\mathrm{Ar}$ & Product & Yield $^{\mathrm{b}}(\%)$ \\
\hline 1 & $\mathrm{H}$ & $\mathrm{Ph}(2 \mathrm{a})$ & $3 a$ & 87 \\
\hline 2 & $\mathrm{H}$ & $4-\mathrm{MeC}_{6} \mathrm{H}_{4}(\mathbf{2 b})$ & 3b & 78 \\
\hline 3 & $\mathrm{H}$ & $3,5-\mathrm{Me}_{2} \mathrm{C}_{6} \mathrm{H}_{3}(2 \mathrm{c})$ & $3 c$ & 74 \\
\hline 4 & $\mathrm{H}$ & $3-\mathrm{MeOC}_{6} \mathrm{H}_{4}(2 \mathrm{~d})$ & $3 d$ & 50 \\
\hline 5 & $\mathrm{H}$ & $4-\mathrm{NCC}_{6} \mathrm{H}_{4}(2 \mathrm{e})$ & $3 e$ & 42 \\
\hline 6 & $\mathrm{H}$ & $4-\mathrm{F}_{3} \mathrm{CC}_{6} \mathrm{H}_{4}(2 \mathrm{f})$ & $3 f$ & 58 \\
\hline 7 & $\mathrm{H}$ & 2-naphthyl (2g) & $3 g$ & 80 \\
\hline 8 & $\mathrm{H}$ & 9-phenanthryl (2h) & $3 h$ & 63 \\
\hline 9 & $\mathrm{H}$ & 4- $\mathrm{PhC}_{6} \mathrm{H}_{4}(2 \mathrm{i})$ & $3 \mathbf{i}$ & 52 \\
\hline 10 & $\mathrm{H}$ & 5-pyrimidyl (2j) & $3 \mathbf{j}$ & 60 \\
\hline 11 & $\mathrm{H}$ & 8-quinolyl (2k) & $3 k$ & 62 \\
\hline 12 & $5-\mathrm{Cl}$ & $\mathrm{Ph}(2 \mathrm{a})$ & 31 & 73 \\
\hline 13 & $5-\mathrm{Cl}$ & $4-\mathrm{MeC}_{6} \mathrm{H}_{4}(\mathbf{2 b})$ & $3 m$ & 67 \\
\hline 14 & $5-\mathrm{Cl}$ & $4-\mathrm{F}_{3} \mathrm{CC}_{6} \mathrm{H}_{4}(2 \mathrm{f})$ & $3 n$ & 57 \\
\hline 15 & $5-\mathrm{NO}_{2}$ & $\mathrm{Ph}(2 \mathrm{a})$ & 30 & 45 \\
\hline 16 & $-\mathrm{c}$ & $4-\mathrm{MeC}_{6} \mathrm{H}_{4}(\mathbf{2 b})$ & $3 p$ & 64 \\
\hline
\end{tabular}

a Reaction conditions: oxazole 1 (0.5 mmol), Cul (20 mol\%), $\mathrm{Fe}_{2} \mathrm{O}_{3}(20$ mol\%), L1 (20 mol\%), LiOt-Bu (1.5 mmol), DTBP (1.0 mmol), arylboronic acid $(1.0 \mathrm{mmol})$, toluene $(3 \mathrm{~mL}), 110^{\circ} \mathrm{C}, 12 \mathrm{~h}$.

b Isolated yield based on 1 after silica gel chromatography.

c The substrate was 4-phenyloxazole.

In view of the results above, substrates were further broadened from 1,3-benzoxazoles to 1,3-benzothiazole (4) and the results are listed in Table 3. From the results we can 
see that our catalytic system is well suited to 1,3-benzothiazole. Similar to the results for 1,3-benzoxazoles $\mathbf{1}$, in the reaction of 1,3-benzothiazole (4) the use of arylboronic acids containing electron-withdrawing groups resulted in a decrease in the yield (entries 5 and 6); the use of arylboronic acids containing electron-donating groups gave only slightly lower yields of product (entries 1-3). Naphthalen2-yl- and phenanthren-9-ylboronic acids also reacted under these conditions in satisfactory yields (entries 7 and 8).

Table 3 Reaction of 1,3-Benzothiazole with Arylboronic Acids ${ }^{\mathrm{a}}$

\begin{tabular}{llll} 
& \\
& & & \\
\hline
\end{tabular}

To gain insight into whether the reaction is a radical reaction or not, a model reaction was carried out with 2,2,6,6-tetramethylpiperidin-1-oxyl (TEMPO, 2.0 equiv) as an additive under standard conditions; the arylation product was obtained with similar yield and no radicals were captured with TEMPO. Referring to previously reported mechanisms, ${ }^{15,20}$ a reaction mechanism was proposed as shown in Scheme 1. Copper(I) iodide is oxidized to copper(II) iodide by iron(III) oxide, which is itself reoxidized by di-tert-butyl peroxide. Then a transmetalation from phenylboronic acid with copper(II) iodide occurred which forms an arylcopper(II) species $\mathbf{A}$. Intermediate $\mathbf{A}$ is oxidized to arylcopper(III) intermediate B. Then, the other transmetalation gives intermediate $\mathbf{C}$. Finally, the desired crosscoupling product is obtained via a reductive elimination reaction.

In summary, an inexpensive and nonpoisonous iron and copper catalytic system has been successfully used, replacing noble metals like palladium salts which are costly and nocuous to the environment, in the reaction of azoles with arylboronic acids. From synthetic point of view, an effective, facile, and environmentally friendly method for the preparation of azoles has been developed.

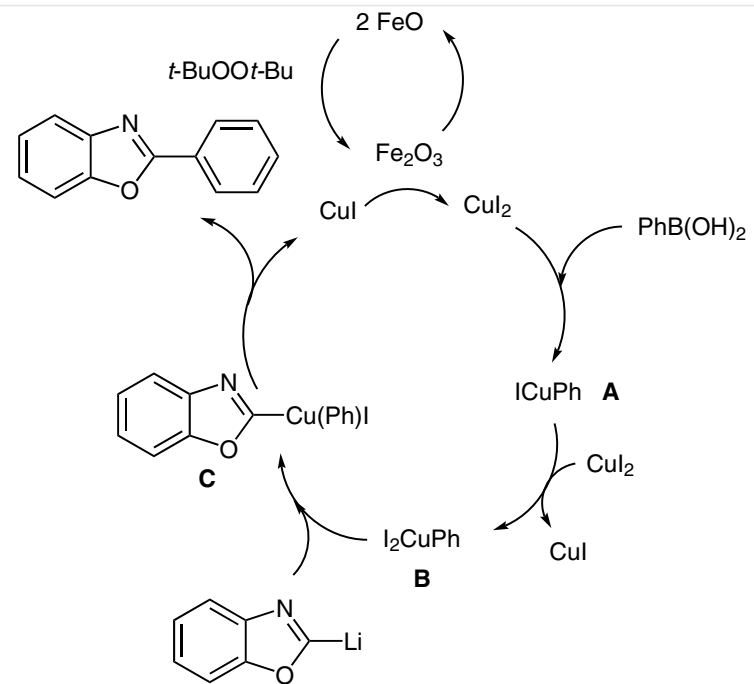

Scheme 1 Proposed reaction mechanism for the iron and copper system catalyzed $\mathrm{C}-\mathrm{H}$ arylation of azoles with arylboronic acids

All reagents and solvents were used directly as obtained commercially unless otherwise noted. Petroleum ether (PE) used refers to the fraction with bp $60-90{ }^{\circ} \mathrm{C}$. Column chromatography was performed with 300-400 mesh silica gel using flash column techniques. ${ }^{1} \mathrm{H}$ and ${ }^{13} \mathrm{C}$ NMR spectra were determined in $\mathrm{CDCl}_{3}$ or DMSO- $d_{6}$ on a VarianInova $400 \mathrm{MHz}$ spectrometer referenced to internal TMS. Chemical shifts in ${ }^{13} \mathrm{C}$ NMR spectra are reported relative to the central line of the $\mathrm{CDCl}_{3}(\delta=77.22)$. IR spectra were recorded on Varian F-1000 spectrometer in $\mathrm{KBr}$. HRMS were obtained with a GCT-TOF instrument.

\section{2-Phenyl-1,3-benzoxazole (3a); ${ }^{12}$ Typical Procedure}

CuI (19 mg, $0.1 \mathrm{mmol}$ ), $\mathrm{Fe}_{2} \mathrm{O}_{3}$ (16 mg, $0.1 \mathrm{mmol}$ ), 1,10-phenanthroline (18 mg, $0.1 \mathrm{mmol}$ ), LiOt-Bu (180 mg, $1.5 \mathrm{mmol}$ ), and di-tert-butyl peroxide (146 mg, $1.0 \mathrm{mmol}$ ) were added to a solution of toluene (3 $\mathrm{mL})$ containing phenylboronic acid $(1.0 \mathrm{mmol})$ and 1,3 -benzoxazole $(0.5 \mathrm{mmol})$. The mixture was stirred at $110^{\circ} \mathrm{C}$ for $12 \mathrm{~h}$. The resulting mixture was then cooled to r.t. and the solvent was removed in vacuo and the remaining residue was purified by column chromatography (silica gel, PE-EtOAc, $20: 1)$ to yield $3 a(84.8 \mathrm{mg}, 87 \%)$ as a light yellow solid; mp $105-107^{\circ} \mathrm{C}$.

IR (KBr): 3060, 1616, 1551, 1489, 1472, 1446, 1343, 1278, 1241, 1052 , $1021,923,807,747,702,687 \mathrm{~cm}^{-1}$.

${ }^{1} \mathrm{H}$ NMR $\left(400 \mathrm{MHz}, \mathrm{CDCl}_{3}\right): \delta=8.23-8.21(\mathrm{~m}, 2 \mathrm{H}), 7.75-7.73(\mathrm{~m}, 1 \mathrm{H})$, 7.55-7.47 (m, $4 \mathrm{H}), 7.32-7.30(\mathrm{~m}, 2 \mathrm{H})$.

${ }^{13} \mathrm{C}$ NMR $\left(100 \mathrm{MHz}, \mathrm{CDCl}_{3}\right): \delta=163.24,150.96,142.30,131.72$, $129.11,127.82,127.36,125.31,124.78,120.22,110.80$.

HRMS (EI): $m / z[M]^{+}$calcd for $\mathrm{C}_{13} \mathrm{H}_{9} \mathrm{NO}$ : 195.0684; found: 195.0685 .

\section{2-p-Tolyl-1,3-benzoxazole (3b) $)^{12}$}

Yellow solid; yield: $81.5 \mathrm{mg}(78 \%)$; $\mathrm{mp} 115-117^{\circ} \mathrm{C}$.

IR (KBr): 3026, 2918, 2306, 1622, 1555, 1501, 1450, 1243, 1177, 1055 , $1017,820,745,726,501 \mathrm{~cm}^{-1}$.

${ }^{1} \mathrm{H}$ NMR $\left(400 \mathrm{MHz}, \mathrm{CDCl}_{3}\right): \delta=8.16(\mathrm{~d}, J=8.0 \mathrm{~Hz}, 2 \mathrm{H}), 7.78-7.71(\mathrm{~m}, 1$ H), 7.59-7.51 (m, $1 \mathrm{H}), 7.36-7.22$ (m, $4 \mathrm{H}), 2.43$ (s, $3 \mathrm{H}$ ). 
${ }^{13} \mathrm{C}$ NMR $\left(100 \mathrm{MHz}, \mathrm{CDCl}_{3}\right): \delta=163.49,150.88,142.37,142.25$, 129.84, 127.79, 125.06, 124.67, 124.60, 120.03, 110.69, 21.85.

HRMS (EI): $m / z$ [M] $]^{+}$calcd for $\mathrm{C}_{14} \mathrm{H}_{11} \mathrm{NO}$ : 209.0841; found: 209.0844 .

\section{2-(3,5-Dimethylphenyl)-1,3-benzoxazole (3c) ${ }^{21}$}

White solid; yield: $82.5 \mathrm{mg}$ (74\%); $\mathrm{mp} 122-124^{\circ} \mathrm{C}$.

IR (KBr): 2916, 2382, 2303, 1600, 1551, 1452, 1243, 1229, 1183, 1003 , $929,863,741,682 \mathrm{~cm}^{-1}$.

${ }^{1} \mathrm{H} \mathrm{NMR}\left(400 \mathrm{MHz}, \mathrm{CDCl}_{3}\right): \delta=7.78(\mathrm{~s}, 2 \mathrm{H}), 7.70-7.61(\mathrm{~m}, 1 \mathrm{H}), 7.46-$ $7.44(\mathrm{~m}, 1 \mathrm{H}), 7.25-7.22(\mathrm{~m}, 2 \mathrm{H}), 7.04(\mathrm{~s}, 1 \mathrm{H}), 2.29(\mathrm{~s}, 6 \mathrm{H})$.

${ }^{13} \mathrm{C}$ NMR (100 MHz, $\left.\mathrm{CDCl}_{3}\right): \delta=163.56,150.82,142.23,138.72$, $133.44,126.99,125.51,125.08,124.63,120.02,110.64,21.38$.

HRMS (EI): $m / z$ [M] $]^{+}$calcd for $\mathrm{C}_{15} \mathrm{H}_{13} \mathrm{NO}$ : 223.0997; found: 223.0998 .

\section{2-(3-Methoxyphenyl)-1,3-benzoxazole (3d) ${ }^{22}$}

White solid; yield: $56.3 \mathrm{mg}(50 \%)$; $\mathrm{mp} 70-72{ }^{\circ} \mathrm{C}$.

IR (KBr): 2833, 2385, 1602, 1555, 1488, 1452, 1243, 1043, 859, 784, $748,725,682 \mathrm{~cm}^{-1}$.

${ }^{1} \mathrm{H} \mathrm{NMR}\left(400 \mathrm{MHz}, \mathrm{CDCl}_{3}\right): \delta=7.98-7.93(\mathrm{~m}, 2 \mathrm{H}), 7.67-7.65(\mathrm{~m}, 1 \mathrm{H})$, 7.46-7.44 (m, $1 \mathrm{H}), 7.30-7.20$ (m, $4 \mathrm{H}), 2.33(\mathrm{~s}, 3 \mathrm{H})$.

${ }^{13} \mathrm{C}$ NMR $\left(100 \mathrm{MHz}, \mathrm{CDCl}_{3}\right): \delta=163.11,160.08,150.89,142.20$, $130.17,128.48,125.34,124.77,120.77,120.17,118.51,112.02$, $110.78,55.68$.

HRMS (EI): $m / z$ [M] $]^{+}$calcd for $\mathrm{C}_{14} \mathrm{H}_{11} \mathrm{NO}_{2}: 225.0790$; found: 225.0795 .

\section{4-(1,3-Benzoxazol-2-yl)benzonitrile (3e) ${ }^{23}$}

White solid; yield: $46.2 \mathrm{mg}$ (42\%); $\mathrm{mp} 204-206^{\circ} \mathrm{C}$.

IR (KBr): 3060, 2227, 1613, 1493, 1474, 1450, 1410, 1341, 1242, 1096, $1055,927,843,816,760,692,548 \mathrm{~cm}^{-1}$.

${ }^{1} \mathrm{H} \mathrm{NMR}\left(400 \mathrm{MHz}, \mathrm{CDCl}_{3}\right): \delta=7.92(\mathrm{~d}, J=8.0,2 \mathrm{H}), 7.64(\mathrm{~d}, J=8.0 \mathrm{~Hz}$, $1 \mathrm{H}), 7.45-7.42$ (m, $2 \mathrm{H}), 7.39-7.31$ (m, $2 \mathrm{H}), 7.23$ (d, J = 7.0 Hz, $1 \mathrm{H}$ ).

${ }^{13} \mathrm{C}$ NMR $\left(100 \mathrm{MHz}, \mathrm{CDCl}_{3}\right): \delta=161.12,151.09,142.05,132.90$, 131.32, 128.17, 126.37, 125.34, 121.72, 120.77, 114.93, 111.09.

HRMS (EI): $m / z$ [M] $]^{+}$calcd for $\mathrm{C}_{14} \mathrm{H}_{8} \mathrm{~N}_{2} \mathrm{O}$ : 220.0637; found: 220.0639 .

\section{2-[4-(Trifluoromethyl)phenyl]-1,3-benzoxazole (3f) $)^{24}$}

White solid; yield: $76.3 \mathrm{mg}$ (58\%); $\mathrm{mp} 154-156{ }^{\circ} \mathrm{C}$.

IR (KBr): 3054, 1917, 1614, 1590, 1482, 1433, 1404, 1321, 1169, 1109, $1064,1011,967,859,754,731,675,617,587 \mathrm{~cm}^{-1}$.

${ }^{1} \mathrm{H}$ NMR $\left(400 \mathrm{MHz}, \mathrm{CDCl}_{3}\right): \delta=8.38(\mathrm{~d}, J=8.12 \mathrm{~Hz}, 2 \mathrm{H}), 7.82-7.80(\mathrm{~m}$, $3 \mathrm{H}), 7.63-7.60(\mathrm{~m}, 1 \mathrm{H}), 7.43-7.38(\mathrm{~m}, 2 \mathrm{H})$.

${ }^{13} \mathrm{C}$ NMR $\left(100 \mathrm{MHz}, \mathrm{CDCl}_{3}\right): \delta=161.70,151.08,142.11,133.37$, $133.04,130.65,128.08,126.15$ ( $\mathrm{q}, J=3.78), 126.0499,125.16,120.62$, 111.02 .

HRMS (EI): $m / z$ [M] $]^{+}$calcd for $\mathrm{C}_{14} \mathrm{H}_{8} \mathrm{NOF}_{3}$ : 263.0558; found: 263.0554 .

\section{2-(Naphthalen-2-yl)-1,3-benzoxazole (3g) ${ }^{12}$}

White solid; yield: $98 \mathrm{mg}(80 \%)$; $\mathrm{mp} 117-119{ }^{\circ} \mathrm{C}$.

IR (KBr): 3050, 2359, 1541, 1452, 1362, 1244, 1178, 1049, 950, 866, $760,750,740,472 \mathrm{~cm}^{-1}$.

$\left.{ }^{1} \mathrm{H} \mathrm{NMR} \mathrm{(400} \mathrm{MHz,} \mathrm{CDCl}_{3}\right): \delta=8.75(\mathrm{~s}, 1 \mathrm{H}), 8.29(\mathrm{~d}, J=8.0 \mathrm{~Hz}, 1 \mathrm{H})$, 7.94 (d, $J=8.0 \mathrm{~Hz}, 2 \mathrm{H}), 7.85-7.79(\mathrm{~m}, 2 \mathrm{H}), 7.55-7.53(\mathrm{~m}, 3 \mathrm{H}), 7.36$ (s, $2 \mathrm{H})$.
${ }^{13} \mathrm{C}$ NMR $\left(100 \mathrm{MHz}, \mathrm{CDCl}_{3}\right): \delta=163.34,151.00,142.36,134.89$, $131.11,129.10,128.93,128.30,128.06,127.95,127.05,125.34$, $124.80,124.52,124.10,120.18,110.76$.

HRMS (EI): $m / z[\mathrm{M}]^{+}$calcd for $\mathrm{C}_{17} \mathrm{H}_{11} \mathrm{NO}$ : 245.0841; found: 245.0839 .

\section{2-(Phenanthren-9-yl)-1,3-benzoxazole (3h) $)^{25}$}

White solid; yield: $92.9 \mathrm{mg}(63 \%) ; \mathrm{mp} 162-164{ }^{\circ} \mathrm{C}$.

IR (KBr): 1615, 1542, 1523, 1447, 1242, 1135, 999, 949, 902, 770, 728 $\mathrm{cm}^{-1}$.

${ }^{1} \mathrm{H}$ NMR $\left(400 \mathrm{MHz}, \mathrm{CDCl}_{3}\right): \delta=9.55-9.52(\mathrm{~m}, 1 \mathrm{H}), 8.80-8.71(\mathrm{~m}, 3 \mathrm{H})$, $8.03(\mathrm{~d}, J=8.0 \mathrm{~Hz}, 1 \mathrm{H}), 7.92-7.89(\mathrm{~m}, 1 \mathrm{H}), 7.77-7.73(\mathrm{~m}, 3 \mathrm{H}), 7.68-$ 7.65 (m, $2 \mathrm{H}), 7.43-7.41$ (m, $2 \mathrm{H})$.

${ }^{13} \mathrm{C}$ NMR $\left(100 \mathrm{MHz}, \mathrm{CDCl}_{3}\right): \delta=162.93,150.40,142.50,131.95$, $131.82,131.01,130.75,130.00,128.92,128.88,127.83,127.34$, $127.32,125.60,124.75,123.15,122.91,122.76,120.54,110.72$.

HRMS (EI): $m / z$ [M] ${ }^{+}$calcd for $\mathrm{C}_{21} \mathrm{H}_{13} \mathrm{NO}$ : 295.0997; found: 295.0994 .

\section{2-(Biphenyl-4-yl)-1,3-benzoxazole (3i) ${ }^{12}$}

White solid; yield: $70.5 \mathrm{mg}$ (52\%); mp $142-143{ }^{\circ} \mathrm{C}$.

IR (KBr): 3027, 1568, 1482, 1446, 1406, 1289, 1245, 1057, 847, 744, $700 \mathrm{~cm}^{-1}$.

${ }^{1} \mathrm{H}$ NMR $\left(400 \mathrm{MHz}, \mathrm{CDCl}_{3}\right): \delta=7.55-7.54(\mathrm{~m}, 2 \mathrm{H}), 7.42-7.35(\mathrm{~m}, 8 \mathrm{H})$, $7.06(\mathrm{~d}, J=8.0 \mathrm{~Hz}, 2 \mathrm{H}), 6.73(\mathrm{~d}, J=8.0 \mathrm{~Hz}, 1 \mathrm{H})$.

${ }^{13} \mathrm{C}$ NMR $\left(100 \mathrm{MHz}, \mathrm{CDCl}_{3}\right): \delta=163.12,150.97,146.76,144.42$, $140.92,128.89,128.28,128.08,127.35,127.00,126.33,125.33$, $124.82,120.16,110.79$.

HRMS (EI): $m / z$ [M] $]^{+}$calcd for $\mathrm{C}_{19} \mathrm{H}_{13} \mathrm{NO}$ : 271.0997; found: 271.0993 .

2-(Pyrimidin-5-yl)-1,3-benzoxazole (3j) $)^{26}$

White solid; yield: $59.1 \mathrm{mg}(60 \%) ; \mathrm{mp} 137-139{ }^{\circ} \mathrm{C}$.

IR (KBr): 3407, 3060, 1549, 1512, 1489, 1458, 1431, 1343, 1216, 1241, $1052,1019,912,824,755,693,675 \mathrm{~cm}^{-1}$.

${ }^{1} \mathrm{H}$ NMR $\left(400 \mathrm{MHz}, \mathrm{CDCl}_{3}\right): \delta=8.17(\mathrm{~s}, 1 \mathrm{H}), 7.97-7.87(\mathrm{~m}, 4 \mathrm{H}), 7.51$ (s, $2 \mathrm{H})$.

${ }^{13} \mathrm{C}$ NMR $\left(100 \mathrm{MHz}, \mathrm{CDCl}_{3}\right): \delta=138.61,133.94,132.86,128.72$, 128.44, 127.88, 126.56, 126.32, 126.21, 125.94.

HRMS (EI): $m / z[\mathrm{M}]^{+}$calcd for $\mathrm{C}_{11} \mathrm{H}_{7} \mathrm{~N}_{3} \mathrm{O}: 197.0589$; found: 197.0586 .

8-(1,3-Benzoxazol-2-yl)quinoline (3k) ${ }^{27}$

Brown oil; yield: $76.3 \mathrm{mg}(62 \%)$.

IR (KBr): 3057, 1654, 1593, 1542, 1494, 1452, 1381, 1320, 1242, 1177 , $1125,1066,924,832,793,746,653,624 \mathrm{~cm}^{-1}$.

${ }^{1} \mathrm{H}$ NMR $\left(400 \mathrm{MHz}, \mathrm{CDCl}_{3}\right): \delta=9.18-9.16(\mathrm{~m}, 1 \mathrm{H}), 8.51-8.49(\mathrm{~m}, 1 \mathrm{H})$, 8.25-8.22 (m, $1 \mathrm{H}), 8.01-7.98(\mathrm{~m}, 1 \mathrm{H}), 7.95-7.92(\mathrm{~m}, 1 \mathrm{H}), 7.69-7.62$ (m, $2 \mathrm{H}), 7.52-7.49(\mathrm{~m}, 1 \mathrm{H}), 7.40-7.37$ ( $\mathrm{m}, 2 \mathrm{H})$.

${ }^{13} \mathrm{C}$ NMR $\left(100 \mathrm{MHz}, \mathrm{CDCl}_{3}\right): \delta=162.37,151.95,151.04,145.97$, $142.39,136.74,132.82,131.83,128.87,126.36,126.09,125.38$, 124.50, 121.81, 120.84, 110.88.

HRMS (EI): $m / z$ [M] $]^{+}$calcd for $\mathrm{C}_{16} \mathrm{H}_{10} \mathrm{~N}_{2} \mathrm{O}: 246.0793$; found: 246.0795 .

\section{5-Chloro-2-phenyl-1,3-benzoxazole (31) ${ }^{28}$}

Yellow solid; yield: $83.6 \mathrm{mg}$ (73\%); $\mathrm{mp} 108-111^{\circ} \mathrm{C}$.

IR (KBr): 3062, 1611, 1553, 1465, 1334, 1262, 1053, 1023, 918, 864, $809,701,683,593 \mathrm{~cm}^{-1}$. 
${ }^{1} \mathrm{H}$ NMR $\left(400 \mathrm{MHz}, \mathrm{CDCl}_{3}\right): \delta=8.17(\mathrm{~d}, J=6.32 \mathrm{~Hz}, 2 \mathrm{H}), 7.68(\mathrm{~s}, 1 \mathrm{H})$, 7.48-7.43 (m, $4 \mathrm{H}), 7.19$ (s, $1 \mathrm{H})$.

${ }^{13} \mathrm{C}$ NMR $\left(100 \mathrm{MHz}, \mathrm{CDCl}_{3}\right): \delta=164.57,149.55,143.35,132.15$, 130.24, 129.21, 127.96, 126.89, 125.59, 120.18, 111.52.

HRMS (EI): $m / z$ [M] ${ }^{+}$calcd for $\mathrm{C}_{13} \mathrm{H}_{8} \mathrm{ClNO}$ : 229.0294; found: 229.0292 .

\section{5-Chloro-2-p-tolyl-1,3-benzoxazole ( $3 \mathrm{~m})^{22}$}

Yellow solid; yield: $81.4 \mathrm{mg}$ (67\%); mp $139-141{ }^{\circ} \mathrm{C}$.

IR (KBr): 2963, 1615, 1559, 1498, 1449, 1260, 1199, 1104, 1016, 916 , $893,792,726,703,498 \mathrm{~cm}^{-1}$.

${ }^{1} \mathrm{H}$ NMR $\left(400 \mathrm{MHz}, \mathrm{CDCl}_{3}\right): \delta=8.12(\mathrm{~d}, J=8.0 \mathrm{~Hz}, 2 \mathrm{H}), 7.73(\mathrm{~d}, J=7.04$ $\mathrm{Hz}, 1 \mathrm{H}), 7.48(\mathrm{~d}, J=8.0 \mathrm{~Hz}, 1 \mathrm{H}), 7.35-7.29(\mathrm{~m}, 3 \mathrm{H}), 2.45(\mathrm{~s}, 3 \mathrm{H})$.

${ }^{13} \mathrm{C}$ NMR $\left(100 \mathrm{MHz}, \mathrm{CDCl}_{3}\right): \delta=168.52,154.28,139.05,132.00$, $129.10,128.17,126.47,125.31,125.05,123.34,121.78,21.54$.

HRMS (EI): $m / z[M]^{+}$calcd for $\mathrm{C}_{13} \mathrm{H}_{10} \mathrm{ClNO}$ : 243.0451; found: 243.0452 .

5-Chloro-2-[4-(trifluoromethyl)phenyl]-1,3-benzoxazole (3n) ${ }^{29}$ White solid; yield: $84.6 \mathrm{mg}$ (57\%); $\mathrm{mp} 147-149^{\circ} \mathrm{C}$.

IR (KBr): 2936, 2359, 1557, 1501, 1453, 1412, 1325, 1261, 1163, 1112, $1072,1014,919,848,806,752,700,589 \mathrm{~cm}^{-1}$.

${ }^{1} \mathrm{H} \mathrm{NMR}\left(400 \mathrm{MHz}, \mathrm{CDCl}_{3}\right): \delta=8.35(\mathrm{~d}, J=8.24 \mathrm{~Hz}, 2 \mathrm{H}), 7.80-7.77(\mathrm{~m}$, $3 \mathrm{H}), 7.53(\mathrm{~d}, J=8.6 \mathrm{~Hz}, 1 \mathrm{H}), 7.35(\mathrm{~m}, 1 \mathrm{H})$.

${ }^{13} \mathrm{C}$ NMR $\left(100 \mathrm{MHz}, \mathrm{CDCl}_{3}\right): \delta=162.97,149.63,143.19,133.40$, 130.66, 130.16, 128.22, 126.33, 126.21 (q, $J=3.69$ ), 125.21, 122.50, $120.55,111.75$.

HRMS (EI): $m / z[M]^{+}$calcd for $\mathrm{C}_{14} \mathrm{H}_{7} \mathrm{ClF}_{3} \mathrm{NO}$ : 297.0168; found: 297.0166.

\section{5-Nitro-2-phenyl-1,3-benzoxazole (3o) ${ }^{29}$}

White solid; yield: $54 \mathrm{mg}$ (45\%); $\mathrm{mp} 167-169{ }^{\circ} \mathrm{C}$.

IR (KBr): 2963, 1615, 1553, 1527, 1449, 1350, 1261, 1067, 1023, 890, $820,736,704,685 \mathrm{~cm}^{-1}$.

${ }^{1} \mathrm{H}$ NMR $\left(400 \mathrm{MHz}, \mathrm{CDCl}_{3}\right): \delta=8.66(\mathrm{~s}, 1 \mathrm{H}), 8.34-8.27(\mathrm{~m}, 3 \mathrm{H}), 7.79$ (d, $J=8.0 \mathrm{~Hz}, 1 \mathrm{H}), 7.62-7.55(\mathrm{~m}, 3 \mathrm{H})$.

${ }^{13} \mathrm{C}$ NMR $\left(100 \mathrm{MHz}, \mathrm{CDCl}_{3}\right): \delta=166.22,154.48,143.15,132.85$, $130.56,129.38,128.27,126.19,121.37,116.52,110.96$.

HRMS (EI): $m / z$ [M] $]^{+}$calcd for $\mathrm{C}_{13} \mathrm{H}_{8} \mathrm{~N}_{2} \mathrm{O}_{3}$ : 240.0535; found: 240.0539 .

\section{4-Phenyl-2-p-tolyloxazole (3p) $)^{30}$}

Yellow solid; yield: $75.2 \mathrm{mg}(64 \%) ; \mathrm{mp} 113-115^{\circ} \mathrm{C}$.

IR (KBr): 3047, 2934, 2418, 1676, 1571, 1483, 1421, 1214, 1165, 1035 , $983,810,733,710,482 \mathrm{~cm}^{-1}$.

${ }^{1} \mathrm{H}$ NMR (400 MHz, DMSO- $d_{6}$ ): $\delta=8.70(\mathrm{~s}, 1 \mathrm{H}), 7.96-7.86(\mathrm{~m}, 4 \mathrm{H})$, 7.46-7.37 (m, $5 \mathrm{H}), 2.38$ (s, $3 \mathrm{H})$.

${ }^{13} \mathrm{C}$ NMR (100 MHz, DMSO- $\left.d_{6}\right): \delta=161.65,141.39,141.19,135.73$, $131.28,130.25,129.32,128.57,126.56,125.73,124.62,21.56$.

HRMS (EI): $m / z$ [M] $]^{+}$calcd for $\mathrm{C}_{16} \mathrm{H}_{13} \mathrm{~N}_{2} \mathrm{O}: 235.0997$; found: 235.0994 .

\section{2-Phenyl-1,3-benzothiazole (5a) $)^{12}$}

Yellow solid; yield: $97 \mathrm{mg}$ (92\%); $\mathrm{mp} 110-113^{\circ} \mathrm{C}$.

IR (KBr): 3061, 1897, 1597, 1306, 1228, 1067, 958, 756, 684, $552 \mathrm{~cm}^{-1}$. ${ }^{1} \mathrm{H} \mathrm{NMR}\left(400 \mathrm{MHz}, \mathrm{CDCl}_{3}\right): \delta=8.17$ (s, $\left.2 \mathrm{H}\right), 7.69$ (s, $\left.1 \mathrm{H}\right), 7.50-7.43$ $(\mathrm{m}, 4 \mathrm{H}), 7.28(\mathrm{~s}, 2 \mathrm{H})$
${ }^{13} \mathrm{C}$ NMR $\left(100 \mathrm{MHz}, \mathrm{CDCl}_{3}\right): \delta=168.21,154.28,135.20,133.75$, 131.12, 129.17, 127.70, 126.47, 125.34, 123.38, 121.77 .

HRMS (EI): $m / z[M]^{+}$calcd for $\mathrm{C}_{13} \mathrm{H}_{9} \mathrm{NS}: 211.0456$; found: 211.0448 .

2-p-Tolyl-1,3-benzothiazole (5b) $)^{12}$

Yellow solid; yield: $93.4 \mathrm{mg}(83 \%) ; \mathrm{mp} 86-89{ }^{\circ} \mathrm{C}$.

IR (KBr): 3056, 2954, 1591, 1447, 1217, 955, 751, $447 \mathrm{~cm}^{-1}$.

${ }^{1} \mathrm{H} \mathrm{NMR}\left(400 \mathrm{MHz}, \mathrm{CDCl}_{3}\right): \delta=8.07(\mathrm{~d}, J=7.72 \mathrm{~Hz}, 2 \mathrm{H}), 7.69-7.67(\mathrm{~m}$, $1 \mathrm{H}), 7.50-7.48(\mathrm{~m}, 1 \mathrm{H}), 7.26(\mathrm{~s}, 4 \mathrm{H}), 2.36(\mathrm{~s}, 3 \mathrm{H})$.

${ }^{13} \mathrm{C}$ NMR $\left(100 \mathrm{MHz}, \mathrm{CDCl}_{3}\right): \delta=168.40,154.33,141.62,135.00$, 131.12, 129.91, 127.66, 126.52, 125.19, 123.22, 121.76, 21.72.

HRMS (EI): $m / z$ [M] $]^{+}$calcd for $\mathrm{C}_{14} \mathrm{H}_{11} \mathrm{NS}$ : 225.0612; found: 225.0618 .

2-(3,5-Dimethylphenyl)-1,3-benzothiazole (5c) $)^{31}$

Yellow solid; yield: $98 \mathrm{mg}$ (82\%); $\mathrm{mp} 72-75^{\circ} \mathrm{C}$.

IR (KBr): 2915, 1773, 1598, 1506, 1432, 1309, 1277, 1184, 1040, 874, $842,754,725,686 \mathrm{~cm}^{-1}$.

${ }^{1} \mathrm{H}$ NMR $\left(400 \mathrm{MHz}, \mathrm{CDCl}_{3}\right): \delta=7.97(\mathrm{~d}, J=8.08 \mathrm{~Hz}, 1 \mathrm{H}), 7.76(\mathrm{~d}, J=$ $7.84 \mathrm{~Hz}, 1 \mathrm{H}), 7.60(\mathrm{~s}, 2 \mathrm{H}), 7.37$ (t, $J=7.53 \mathrm{~Hz}, 1 \mathrm{H}), 7.25(\mathrm{t}, J=7.42 \mathrm{~Hz}$, $1 \mathrm{H}), 7.00(\mathrm{~s}, 1 \mathrm{H}), 2.29(\mathrm{~s}, 6 \mathrm{H})$.

${ }^{13} \mathrm{C}$ NMR $\left(100 \mathrm{MHz}, \mathrm{CDCl}_{3}\right): \delta=168.99,154.22,138.84,135.11$, $133.54,132.90,126.38,125.47,125.18,123.22,121.71,21.38$.

HRMS (EI): $m / z[M]^{+}$calcd for $\mathrm{C}_{14} \mathrm{H}_{13} \mathrm{NS}$ : 239.0769; found: 239.0766 .

2-(3-Methoxyphenyl)-1,3-benzothiazole (5d) ${ }^{32}$

White solid; yield: $54.2 \mathrm{mg}$ (45\%); $\mathrm{mp} 84-87^{\circ} \mathrm{C}$.

IR (KBr): 3060, 2833, 1604, 1470, 1289, 1047, 995, 871, 761, 727, 684 $\mathrm{cm}^{-1}$.

${ }^{1} \mathrm{H}$ NMR $\left(400 \mathrm{MHz}, \mathrm{CDCl}_{3}\right): \delta=8.07(\mathrm{~d}, J=8.04,1 \mathrm{H}), 7.88(\mathrm{~d}, J=7.84$ $\mathrm{Hz}, 1 \mathrm{H}), 7.64(\mathrm{~m}, 2 \mathrm{H}), 7.48(\mathrm{t}, J=7.64, \mathrm{~Hz}, 1 \mathrm{H}), 7.39-7.35(\mathrm{~m}, 2 \mathrm{H})$, $7.02(\mathrm{~d}, J=7.56 \mathrm{~Hz}, 1 \mathrm{H}), 3.89(\mathrm{~s}, 3 \mathrm{H})$.

${ }^{13} \mathrm{C}$ NMR $\left(100 \mathrm{MHz}, \mathrm{CDCl}_{3}\right): \delta=168.08,160.10,154.20,135.23$, $135.03,130.18,126.46,125.37,123.38,121.76,120.37,117.47$, $112.17,55.63$.

HRMS (EI): $m / z$ [M] ${ }^{+}$calcd for $\mathrm{C}_{14} \mathrm{H}_{11}$ NOS: 241.0561; found: 241.0556 .

\section{4-(1,3-Benzothiazol-2-yl)benzonitrile (5e) ${ }^{25}$}

White solid; yield: $43.7 \mathrm{mg}$ (37\%); $\mathrm{mp} 163-165{ }^{\circ} \mathrm{C}$.

IR (KBr): 3062, 2227, 1603, 1495, 1476, 1450, 1412, 1343, 1245, 1061 , $834,679,618,553 \mathrm{~cm}^{-1}$.

${ }^{1} \mathrm{H}$ NMR $\left(400 \mathrm{MHz}, \mathrm{CDCl}_{3}\right): \delta=7.85(\mathrm{~d}, J=8.44 \mathrm{~Hz}, 2 \mathrm{H}), 7.57(\mathrm{~d}, J=$ $8.20 \mathrm{~Hz}, 1 \mathrm{H}), 7.36(\mathrm{~d}, J=8.44 \mathrm{~Hz}, 2 \mathrm{H}), 7.31-7.24(\mathrm{~m}, 2 \mathrm{H}), 7.16(\mathrm{~d}, J=$ $7.04 \mathrm{~Hz}, 1 \mathrm{H})$.

${ }^{13} \mathrm{C}$ NMR $\left(100 \mathrm{MHz}, \mathrm{CDCl}_{3}\right): \delta=146.91,138.70,133.35,132.49$, 129.82, 129.14, 128.95, 126.86, 119.17, 118.40, 111.94, 100.48 .

HRMS (EI): $m / z[\mathrm{M}]^{+}$calcd for $\mathrm{C}_{14} \mathrm{H}_{8} \mathrm{~N}_{2} \mathrm{~S}: 241.0561$; found: 241.0556 .

2-[4-(Trifluoromethyl)phenyl]-1,3-benzothiazole (5f) $)^{25}$

White solid; yield: $76.7 \mathrm{mg}$ (55\%); mp 161-163 ${ }^{\circ} \mathrm{C}$.

IR (KBr): 3056, 1919, 1617, 1484, 1435, 1407, 1323, 1173, 1109, 1066, $971,842,760,678,620,589 \mathrm{~cm}^{-1}$.

${ }^{1} \mathrm{H}$ NMR $\left(400 \mathrm{MHz}, \mathrm{CDCl}_{3}\right): \delta=8.18(\mathrm{~d}, J=8.0 \mathrm{~Hz}, 2 \mathrm{H}), 8.09(\mathrm{~d}, J=8.0$ $\mathrm{Hz}, 1 \mathrm{H}), 7.90(\mathrm{~d}, J=8.0 \mathrm{~Hz}, 1 \mathrm{H}), 7.73(\mathrm{~d}, J=8.0 \mathrm{~Hz}, 2 \mathrm{H}), 7.51(\mathrm{t}, J=8.0$ $\mathrm{Hz}, 1 \mathrm{H}), 7.41(\mathrm{t}, J=8.0 \mathrm{~Hz}, 1 \mathrm{H})$. 
${ }^{13} \mathrm{C}$ NMR $\left(100 \mathrm{MHz}, \mathrm{CDCl}_{3}\right): \delta=166.26,154.25,136.98,135.41$, 132.50, 127.98, 126.87, 126.23 (q, J = 3.7), 126.00, 123.84, 121.96.

HRMS (EI): $m / z$ [M] $]^{+}$calcd for $\mathrm{C}_{14} \mathrm{H}_{8} \mathrm{~F}_{3} \mathrm{NS}: 279.0330$; found: 279.0332 .

\section{2-(Naphthalen-2-yl)-1,3-benzothiazole (5g) ${ }^{12}$}

Yellow solid; yield: $94 \mathrm{mg}$ (72\%); $\mathrm{mp} 126-128^{\circ} \mathrm{C}$.

IR (KBr): 3045, 2347, 1527, 1442, 1353, 1224, 1164, 1030, 939, 857, $749,737,726,459 \mathrm{~cm}^{-1}$.

${ }^{1} \mathrm{H} \mathrm{NMR}\left(400 \mathrm{MHz}, \mathrm{CDCl}_{3}\right): \delta=8.57(\mathrm{~s}, 1 \mathrm{H}), 8.22(\mathrm{~d}, J=8.0 \mathrm{~Hz}, 1 \mathrm{H})$, $8.12(\mathrm{~d}, J=8.0 \mathrm{~Hz}, 1 \mathrm{H}), 7.99-7.93(\mathrm{~m}, 3 \mathrm{H}), 7.90-7.88(\mathrm{~m}, 1 \mathrm{H}), 7.57-$ $7.54(\mathrm{~m}, 2 \mathrm{H}), 7.51(\mathrm{~d}, J=8.0 \mathrm{~Hz}, 1 \mathrm{H}), 7.41(\mathrm{t}, J=8.0 \mathrm{~Hz}, 1 \mathrm{H})$.

${ }^{13} \mathrm{C}$ NMR $\left(100 \mathrm{MHz}, \mathrm{CDCl}_{3}\right): \delta=168.34,154.43,135.32,134.81$, $133.39,131.18,129.04,128.08,127.79,127.68,127.10,126.60$, 125.46, 124.64, 123.44, 122.24, 121.86 .

HRMS (EI): $m / z$ [M] $]^{+}$calcd for $\mathrm{C}_{17} \mathrm{H}_{11} \mathrm{NS}$ : 261.0612; found: 261.0615 .

\section{2-(Phenanthren-9-yl)-1,3-benzothiazole $(5 \mathrm{~h})^{33}$}

Yellow oil; yield: $91.7 \mathrm{mg}$ (59\%).

IR (KBr): 1627, 1554, 1533, 1450, 1254, 1147, 1012, 958, 921, 787, $741 \mathrm{~cm}^{-1}$.

${ }^{1} \mathrm{H} \mathrm{NMR}\left(400 \mathrm{MHz}, \mathrm{CDCl}_{3}\right): \delta=8.92(\mathrm{~d}, J=8.0 \mathrm{~Hz}, 1 \mathrm{H}), 8.79(\mathrm{~d}, J=8.0$ $\mathrm{Hz}, 1 \mathrm{H}), 8.74(\mathrm{~d}, J=8.0 \mathrm{~Hz}, 1 \mathrm{H}), 8.22(\mathrm{~d}, J=8.0 \mathrm{~Hz}, 2 \mathrm{H}), 7.99(\mathrm{t}, J=8.0$ $\mathrm{Hz}, 2 \mathrm{H}), 7.77-7.64(\mathrm{~m}, 4 \mathrm{H}), 7.58(\mathrm{t}, J=8.0 \mathrm{~Hz}, 1 \mathrm{H}), 7.48(\mathrm{t}, J=8.0 \mathrm{~Hz}$, $1 \mathrm{H})$.

${ }^{13} \mathrm{C}$ NMR $\left(100 \mathrm{MHz}, \mathrm{CDCl}_{3}\right): \delta=167.78,154.28,135.58,131.37$, $131.25,131.01,130.86,130.01,129.60,129.42,128.37,127.66$, $127.42,127.31,126.89,126.54,125.60,123.78,123.10,122.90$, 121.63.

HRMS (EI): $m / z$ [M] $]^{+}$calcd for $\mathrm{C}_{21} \mathrm{H}_{13} \mathrm{NS}$ : 211.0769; found: 211.0771 .

\section{Acknowledgment}

We gratefully acknowledge A Project Funded by the Priority Academic Program Development of Jiangsu Higher Education Institutions, The Project of Scientific and Technologic Infrastructure of Suzhou (SZS201207) and the National Natural Science Foundation of China (No. 21072143) for financial support.

\section{Supporting Information}

Supporting information for this article is available online at http://dx.doi.org/10.1055/s-0034-1379073.

\section{References}

(1) (a) Tzanopoulou, S.; Sagnou, M.; Paravatou-Petsotas, M.; Gourni, E.; Loudos, G.; Xanthopoulos, S.; Lafkas, D.; Kiaris, H.; Varvarigou, A.; Pirmettis, I. C.; Papadopoulos, M.; Pelecanou, M. J. Med. Chem. 2010, 53, 4633. (b) Van Zandt, M. C.; Jones, M. L.; Gunn, D. E.; Geraci, L. S.; Jones, J. H.; Sawicki, D. R.; Sredy, J.; Jacot, J. L.; DiCioccio, A. T.; Petrova, T.; Mitschler, A.; Podjarny, A. D. J. Med. Chem. 2005, 48, 3141. (c) Katz, L. J. Am. Chem. Soc. 1953, 75, 712. (d) Dalvie, D. K.; Kalgutkar, A. S.; KhojastehBakht, S. C.; Obach, R. S.; O'Donnell, J. P. Chem. Res. Toxicol. 2002, 15, 269. (e) Boeini, H. Z.; Najafabadi, K. H. Eur. J. Org. Chem. 2009, 4926.
(2) (a) Riadi, Y.; Mamouni, R.; Azzalou, R.; El Haddad, M.; Routier, S.; Guillaumet, G.; Lazar, S. Tetrahedron Lett. 2011, 52, 3492. (b) Wang, H.; Wang, L.; Shang, J.; Li, X.; Wang, H.; Gui, J.; Lei, A. Chem. Commun. 2012, 48, 76. (c) Bonnamour, J.; Bolm, C. Org. Lett. 2008, 10, 2665. (d) Yuan, Y.; Thomé, I.; Kim, S. H.; Chen, D.; Beyer, A.; Bonnamour, J.; Zuidema, E.; Chang, S.; Bolm, C. Adv. Synth. Catal. 2010, 352, 2892.

(3) Lee, D.-H.; Choi, M.; Yu, B.-W.; Ryoo, R.; Taher, A.; Hossain, S.; Jin, M.-J. Adv. Synth. Catal. 2009, 351, 2912.

(4) (a) Miyaura, N.; Suzuki, A. Chem. Rev. 1995, 95, 2457. (b) Littke, A. F.; Fu, G. C. Angew. Chem. Int. Ed. 2002, 41, 4176.

(5) Shibahara, F.; Yamaguchi, E.; Murai, T. Chem. Commun. 2010, 46, 2471.

(6) Huang, J.; Chan, J.; Chen, Y.; Borths, C. J.; Baucom, K. D.; Larsen, R. D.; Faul, M. M. J. Am. Chem. Soc. 2010, 132, 3674.

(7) Do, H.-Q.; Daugulis, O.J. Am. Chem. Soc. 2007, 129, 12404.

(8) Sezen, B.; Sames, D. Org. Lett. 2003, 5, 3607.

(9) Zhang, W.; Zeng, Q.; Zhang, X.; Tian, Y.; Yue, Y.; Guo, Y.; Wang, Z. J. Org. Chem. 2011, 76, 4741.

(10) Guchhait, S. K.; Kashyap, M.; Saraf, S. Synthesis 2010, 1166.

(11) Hachiya, H.; Hirano, K.; Satoh, T.; Miura, M. ChemCatChem 2010, 2, 1403.

(12) Ranjit, S.; Liu, X. Chem. Eur. J. 2011, 17, 1105.

(13) Wang, H.; Wang, Y.; Peng, C.; Zhang, J.; Zhu, Q. J. Am. Chem. Soc. 2010, 132, 13217.

(14) Li, Z.; Cui, Z.; Liu, Z.-Q. Org. Lett. 2013, 15, 406.

(15) Yang, F.; Xu, Z.; Wang, Z.; Yu, Z.; Wang, R. Chem. Eur. J. 2011, 17, 6321.

(16) Liu, X.; Zhang, S. Synlett 2011, 268.

(17) Liu, X.; Zhang, S. Synlett 2011, 1137.

(18) Zhang, S.; Liu, X.; Wang, T. Adv. Synth. Catal. 2011, 353, 1463.

(19) Wang, P.; Liu, X.; Zhang, S. Chin. J. Chem. 2013, 31, 187.

(20) (a) King, A. E.; Brunold, T. C.; Stahl, S. S. J. Am. Chem. Soc. 2009, 131, 5044. (b) Ribas, X.; Calle, C.; Poater, A.; Casitas, A.; Gómez, L.; Xifra, R.; Parella, T.; Benet-Buchholz, J.; Schweiger, A.; Mitrikas, G.; Solà, M.; Llobet, A.; Stack, T. D. P. J. Am. Chem. Soc. 2010, 132, 12299. (c) Casitas, A.; King, A. E.; Parella, T.; Costas, M.; Stahl, S. S.; Ribas, X. Chem. Sci. 2010, 1, 326.

(21) Ackermann, L.; Althammer, A.; Fenner, S. Angew. Chem. Int. Ed. 2009, 48, 201.

(22) Ackermann, L.; Barfüsser, S.; Pospech, J. Org. Lett. 2010, 12, 724.

(23) Blacker, A. J.; Farah, M. M.; Hall, M. I.; Marsden, S. P.; Saidi, O.; Williams, J. M. J. Org. Lett. 2009, 11, 2039.

(24) Xing, R.-G.; Li, Y.-N.; Liu, Q.; Meng, Q.-Y.; Li, J.; Shen, X.-X.; Liu, Z.; Zhou, B.; Yao, X.; Liu, Z.-L. Eur. J. Org. Chem. 2010, 6627.

(25) Hachiya, H.; Hirano, K.; Satoh, T.; Miura, M. Org. Lett. 2009, 11, 1737.

(26) Bayh, O.; Awad, H.; Mongin, F.; Hoarau, C.; Bischoff, L.; Trécourt, F.; Quéguiner, G.; Marsais, F.; Blanco, F.; Abarca, B.; Ballesteros, R. J. Org. Chem. 2005, 70, 5190.

(27) Laroche, J.; Beydoun, K.; Guerchais, V.; Doucet, H. Catal. Sci. Technol. 2013, 3, 2072.

(28) Lee, J. J.; Kim, J.; Jun, Y. M.; Lee, B. M.; Kim, B. H. Tetrahedron 2009, 65, 8821.

(29) Zhang, M.; Zhang, S.; Liu, M.; Cheng, J. Chem. Commun. 2011, 47, 11522.

(30) Flegeau, E. F.; Popkin, M. E.; Greaney, M. F. Org. Lett. 2006, 8, 2495.

(31) Saha, D.; Adak, L.; Ranu, B. C. Tetrahedron Lett. 2010, 51, 5624.

(32) Inamoto, K.; Hasegawa, C.; Hiroya, K.; Doi, T. Org. Lett. 2008, 10 , 5147.

(33) Yamamoto, T.; Muto, K.; Komiyama, M.; Canivet, J.; Yamaguchi, J.; Itami, K. Chem. Eur. J. 2011, 17, 10113. 